\title{
Development of a drive management system by V2I communication
}

\author{
Alexandra Elisabeta Lörincz ${ }^{1, *}$, Marius Nicolae Risteiu ${ }^{1}$, and Alin Constandoiu ${ }^{1}$ \\ ${ }^{1}$ University of Petrosani, Departament of Computer and Electrical Engineering, Romania
}

\begin{abstract}
In this paper we propose a solution for drive management, by communicating between the vehicles involved in traffic and also with the road infrastructure, like traffic lights, road signs etc. All these elements make up a network in which information sharing is very quick and very safe. This system only warns the driver of dangers or some information that he missed on the road, like road signs. The system does not take control of the vehicle, but displays on an LCD display warnings, information on the operation of the car's engine (information received from the ECU) and information about the distance to the other cars around him.
\end{abstract}

\section{Introduction}

The continuous development in the automotive domain, lead to the implementation of new systems and technologies for the benefit of drivers but also for the passengers in vehicle. [1]

These systems are designed for specific reasons that are based on well-defined road traffic safety, easier management of vehicles by people and avoiding damage to property which greatly affects the economy because of accidents.

Avoiding these dangers led to development of certain systems center who warns the driver about a possible impact with another vehicle, pedestrian or cyclist but also warning the exit in cases unintentional vehicle off the road and an accident. These systems have been implemented even by some automobile manufacturers and the most advanced technologies include acquiring control of the vehicle in cases of extreme danger or when the driver announced this (setting automatic mode driving the car).[2]

To implement these systems on vehicles, manufacturers have developed specific communication networks between vehicles that allow transmission to other cars of basic information such as speed, direction of travel, exact location where there are vehicle messages to avoid traffic jams traffic, and weather information on a particular route.

This exchange of information made through various methods: radio communications, wireless communications, GSM communications or communications through satellites.[3]

Creating this system begins with the hardware, which includes radio module (RFM), module Wi-Fi (wireless), sensors etc. but the most important part is the software that applies a specific protocol that system. Protocol development allows us communication

"Corresponding author: lorinczalexandra@yahoo.com 
between vehicles but also communication infrastructure that includes road traffic lights, road signs, etc.

All these elements make up a network in which information sharing is very quick and very safe. This system only warns the driver of dangers or some information that you do not know, he did not see or did not want to see them! (Road signs). The system not only takes control of the vehicle on an LCD display sends warnings, information on the operation of the car's engine (information received from the ECU) and information about the distance that has over other cars around him.

This system is created from devices placed on road signs and in-vehicle devices that creates a network and produce a useful exchange of information between them. [4]

\subsection{Communication between vehicles and road infrastructure V2I}

This communication between drivers and road infrastructure is beneficial in several ways:

- $\quad$ receiving information about road / highway which is the vehicle at the time;

- information on the maximum and minimum travel speed on certain portions of the road. This information is very useful for a leader traveling an unknown road or the possibility of another traffic law in some countries.

- $\quad$ information on dangerous roads with high accident

- $\quad$ heavy traffic in large cities who own many traffic lights and road signs

- $\quad$ informing the driver on the points of interest that are nearby.

Road infrastructure represents all roads usually open to public traffic, with road signs and decorations, that a person can move a vehicle from point A to point B. [5]

Romanian legislation requires specializing cataloging roads in three categories in the following Table1:

Table 1. The traffic signs

\begin{tabular}{|c|c|c|c|}
\hline From a legal perspective & & & Traffic signs \\
\hline \multirow[t]{2}{*}{ The destination } & $\begin{array}{l}\text { Private utility } \\
\text { roads }\end{array}$ & & \\
\hline & Public roads & & \\
\hline \multirow[t]{3}{*}{ The movement } & $\begin{array}{l}\text { Open to public } \\
\text { traffic roads }\end{array}$ & & \multirow{3}{*}{$\begin{array}{l}66 \text { Transfăgăraşan } \\
\begin{array}{|l}\text { ÎNCHIS } \\
\text { deschis } \\
\text { pâna la : } \\
\text { Bâlea Cascadă }\end{array}\end{array}$} \\
\hline & \multirow[t]{2}{*}{$\begin{array}{l}\text { Close to public } \\
\text { traffic roads }\end{array}$} & & \\
\hline & & & \\
\hline \multirow[t]{6}{*}{$\begin{array}{l}\text { Functional and } \\
\text { administrative territorial }\end{array}$} & \multirow[t]{3}{*}{$\begin{array}{l}\text { National interest } \\
\text { roads }\end{array}$} & Highways & \\
\hline & & Express roads & E40 \\
\hline & & National roads & $\widehat{65}$ \\
\hline & $\begin{array}{l}\text { County interest } \\
\text { roads }\end{array}$ & County roads & $100 A$ \\
\hline & \multirow{2}{*}{$\begin{array}{l}\text { Local interes } \\
\text { roads }\end{array}$} & Village roads & 169 \\
\hline & & Streets & \\
\hline
\end{tabular}


All these road signs are classified in eight specific categories:

- Road signs Warning: This category contains 59 different indicators;

- Road signs Priority: This category contains 6 road signs;

- Road signs Banning and restriction: This category contains 40 road signs;

- Road signs Ordering: This category contains 12 indicators;

- Road signs Guiding : This category contains 35 indicators;

- Road signs Information: This category contains 48 indicators;

- Road signs Tourist Information: This category contains 12 ;

- Road signs, additional panels: contains 23 indicators.

Each road sign includes one special device designed, to communicate with vehicles moving or stationary, information about its specification, Fig. 1.

All this communication is performed using a specific protocol created for this purpose.

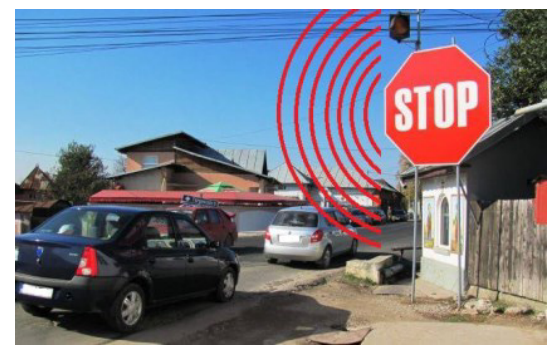

Fig. 1. Comunication V2I [6]

\section{$1.2 \mathrm{~V} 2 \mathrm{~V}$ communication protocol}

Department of Transport (DOT) of the United States compel car makers to include communications systems "vehicle-to-vehicle '(V2V) for reducing the number of road accidents.

$\mathrm{V} 2 \mathrm{~V}$ communication technology consists of motor vehicles between them by changing information useful in avoiding / preventing some accidents, but also early warning of the potential dangers of driver in traffic. This communication between vehicles is made using radio waves which $\mathrm{I}$ hope is opposed to cameras or certain sensors have a range of about 300 meters and are not blocked by some object in their area or another cars. Using this technology can prevent the driver from the dangers of traffic especially at intersections or when changing lane movement and travel.

V2V is also known as the VANETs (Vehicular Ad Hoc Networks). In 2001, it was mentioned in a publication as ad hoc networks can be formed by cars, which can help avoid blind spots, avoiding accidents etc. [7]

All this communication is performed using radio networks using special modules for sending and receiving data.

\section{Proposed V2V and V2I communication protocol}

This protocol is designed to warn the driver about road signs around it, the information you read in real time from the onboard computer of the vehicle on K-Line protocol and information on vehicles around it.

In Fig. 2 is present a block diagram of communication V2V and V2I. 


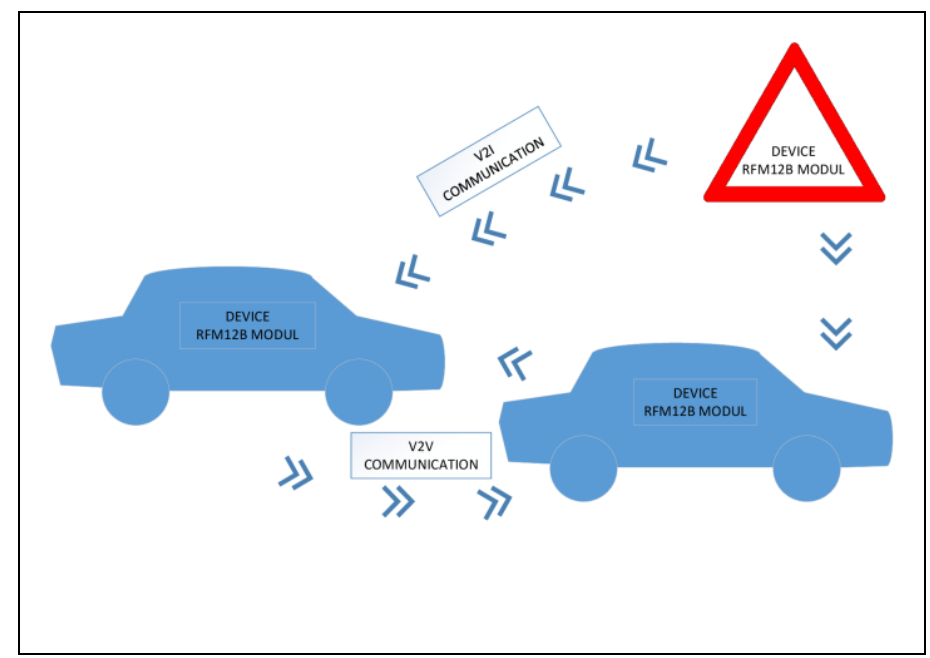

Fig. 2. Communication V2V and V2I.

\subsection{Hardware System}

The device of data transmission by moving or stationary vehicles, and road sign is placed on the sending data every second.

The module is use of a data transmission radio module (RFM12B) operating at a frequency of $915 \mathrm{MHz}$, at a distance of $250 \mathrm{~m}$ in the open air.

It creates STM32 with the microcotroller communication device, Fig.3.

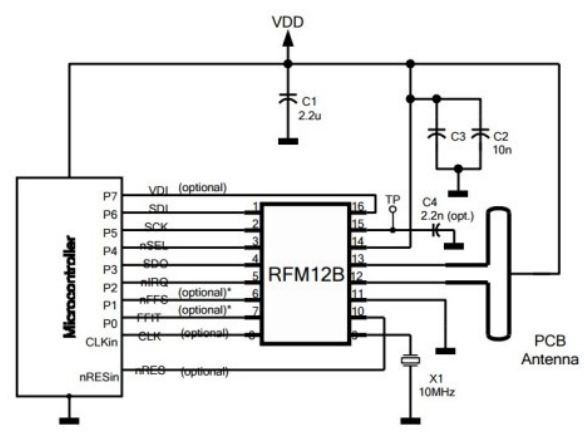

Fig. 3. Typical application with RFM12B.

Inside the vehicle is located this device RFM12B, set in slave mode and communicates with another vehicle (or more vehicles) which in turn holds the device (V2V communication) or communicate with road infrastructure (traffic signs, traffic lights etc.) holding devices RFM12B set in Master mode (V2I communication).

All the communication between the microcontroller and RFM12B module is done with SPI serial interface.

The Serial Peripheral Interface bus (SPI) is a synchronous serial communication interface specification used for short distance communication, primarily in embedded systems.

SPI devices communicate in full duplex mode using a master-slave architecture with a single master. The master device originates the frame for reading and writing. Multiple slave devices are supported through selection with individual slave select (SS) lines. [8] 


\subsection{Software System}

Data sent from road signs to vehicles or directly between vehicles, forming a communication network where road signs are "Master", they only send data objects around them, and the vehicles are and "Master" and "Slave", they send priority their data and receive data from road signs or vehicles around them.

All this data is continuously exchange data and real-time data is received and transmitted between traffic participants.

The microcontroller communicates via SPI interface (Serial- Peripheral Interface) module RFM12B, which is a high speed serial interface that operates in full duplex mode.

The data stream transmitted automatically be filled with information specific vehicle or road sign.

This raw data consists of more bytes owning a priority and have a specific meaning if inficator received from a road or a vehicle.

In the next figure is presented the initialization function for RFM12B written in the microcontroller program (Fig.4).

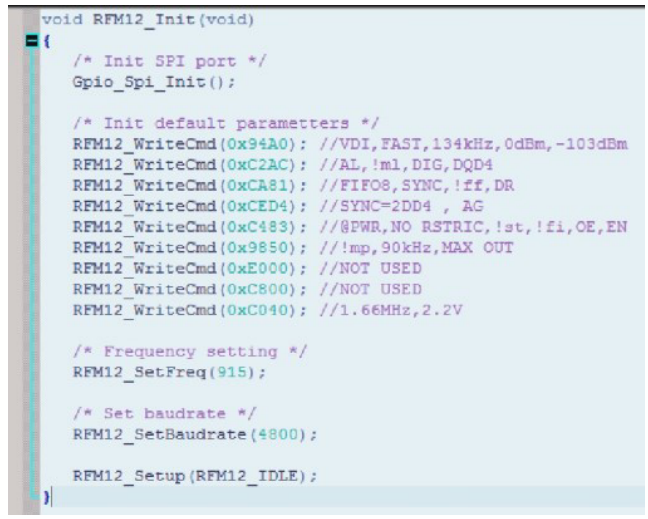

Fig. 4. Initializing communication with RFM12B module.

\subsection{Message structure}

The message structure is composed of header bytes, 1 byte for data length, 8 bytes of data, a checksum byte and 1 bytes stop.

Start Bytes to announce the receipt of an indicator, traffic lights or other vehicles of a new data message.

Stop Byte announces completion of the string received.

Bytes of data are divided into several categories.

- The first byte signify that comes directly from this message. It may be received from a road sign, a traffic light or in another vehicle. First Byte has the highest priority in continuing reading data from that string data.

- Second byet holds that a value according to the first byte. This value can range from 0 8 (warning signs, signs priority etc), where the first byte is the meaning of road signs.

- Third byte value has specific category of which the second byte. This category may vary from $0 \sim 59$.

- Byte 4 has the same principles as the first byte of data. If there are two signs in the same place, they can communicate this to us. 4 'lea byte can have the value of expressing an indicator, a traffic light or another vehicle.

- Byte 5 has the same features with byte No. 2 . 
- Byte 6 is strictly related to byte 5 and its value is specific category which includes byte 5.

- Byte 7 and 8 are used strictly for communication between vehicles, and mean values of speed or their location.

The checksum byte (CS) inserted at the end of the message block is defined as the simple 8 bit sum series of all the bytes in the message, excluding the checksum. [9]

The CS calculation is as follows:

If the message is:

$<1><2><3>\ldots<\mathrm{N}>,<\mathrm{CS}>$

Where $<\mathrm{i}>(1<=\mathrm{i}<=\mathrm{N})$ is the numeric value of the ith byte of message, then:

$<\mathrm{CS}>=<\mathrm{CS}>\mathrm{N}$

Where $<\mathrm{CS}>\mathrm{i}(\mathrm{i}=2$ to $\mathrm{N})$ is defined as

$<\mathrm{CS}>\mathrm{i}=\{<\mathrm{CS}>\mathrm{i}-1+<\mathrm{i}>\}$ Modulo 256

And $<\mathrm{CS}>1=<1>$

Table 2. Message structure

\begin{tabular}{|l|l|l|}
\hline \multicolumn{1}{|c|}{ Sequence } & \multicolumn{1}{c|}{ Description } & \multicolumn{1}{c|}{ Data } \\
\hline Byte 1 & Message header 1 & 0xAA \\
\hline Byte 2 & Message header 2 & 0xAA \\
\hline Byte 3 & Message header 3 & 0x2D \\
\hline Byte 4 & Message header 4 & 0xD4 \\
\hline Byte 5 & Message length & 0x08 \\
\hline Bytes 6 to 13 & Data (up to 7 bytes) & 0xXX \\
\hline Byte 14 & Message stop 1 & 0xAA \\
\hline Checksum Byte 15 & Checksum & 0xYY \\
\hline
\end{tabular}

To send a message using radio module RFM12B is need for some specific command this (Transmitter Register Write Command).

Table 3. Message block - Transmitter Register Write Command.

\begin{tabular}{|l|l|l|l|l|l|l|l|l|l|l|l|l|l|l|l|l|}
\hline Bit & 15 & 14 & 13 & 12 & 11 & 10 & 9 & 8 & 7 & 6 & 5 & 4 & 3 & 2 & 1 & 0 \\
\hline 0xB8AA & 1 & 0 & 1 & 1 & 1 & 0 & 0 & 0 & $\mathrm{t} 7$ & $\mathrm{t} 6$ & $\mathrm{t} 5$ & $\mathrm{t} 4$ & $\mathrm{t} 3$ & $\mathrm{t} 2$ & $\mathrm{t} 1$ & $\mathrm{t} 0$ \\
\hline
\end{tabular}

With this command, the controller can write 8 bits ( $\mathrm{t} 7$ to $\mathrm{t} 0$ ) to the transmitter data register. Bit 7 (el) must be set in Configuration Setting Command. [10]

An example of sending data from one road sign specified in the following figure, where SPI (Serial Peripheral Interface) has the following characteristics:

- MSB (Most Significant Bit) - First,

- 8 bit transfer,

- clock is low when inactive $\mathrm{CPOL}=0$,

- data is valid on clock leading edge $\mathrm{CPHA}=0$,

- enable line is active low. 


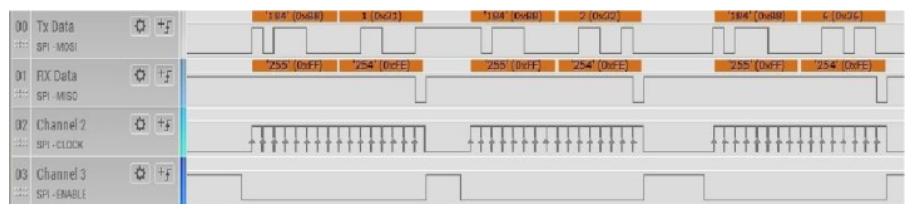

Fig. 5. The signals sent by the traffic signs.

The communication between the microcontroller and module RFM12B is performed using the SPI interface (Serial Peripheral Interface).

In Fig. 5 are shown data signals:

- SPI MOSI - Master Out Slave In (transmit data from Master device to Slave device)

- SPI MISO - Master In Slave Out (receive data to Master device from Slave device)

- SPI CLOCK - Serial clock (from the Master device)

- SPI ENABLE - Slave select (active low, out of Master device)

First byte $0 \times B 8$ and $0 \times 31$, signify that the message is from a traffic sign, the second byte $0 \times \mathrm{xB} 8$ with $0 \times 32$ signify category traffic sign warning and the third byte $0 \times \mathrm{xB} 8$ with $0 \times 36$ signifies (the narrow road on the left).

The result of calculation described of the message is Checksum 0xA5.

After receiving this message by vehicle, the LCD will show the road sign specified.

\section{Conclusions}

This data communication helps alert the drivers from time with information about traffic, road signs, etc.

Composing hardware part is very simple, with a very low cost and easy to implement in any car.

V2V communication system proves to be the best system to achieve data transfer and information (operating at a frequency of $915 \mathrm{MHz}$ ) compared with systems of vehicles carried by cellular networks, GPS etc. so is a high degree of flexibility for implementation. This communication is not restricted to different categories of roads or geographic areas (as restricting communication systems based on GSM or GPS).

Communication is done at a maximum distance of 250 meters between two cars in the open air.

In crowded cities are many factors that prevent communication to the maximum distance (buildings, trees, etc.), this can turn the disadvantage into an advantage, because speed is much smaller and the need to interpret information at a large distance is not necessary.

\section{References}

1. R. Dovleac, A. Ionica, M. Leba, Proceedings of Review of Management and Economic Engineering, 32-39 (2016)

2. https://www.nhtsa.gov/sites/nhtsa.dot.gov/files/readiness-of-v2v-technology-for-application812014.pdf

3. www.termo.utcluj.ro/confstud07/lucrari/socea.doc

4. A. Ionica, M. Leba, Procedia Economics and Finances, 671-676, 23 (2015)

5. http://infrastructura-rutiera.blogspot.ro/

6. http://adevarul.ro/locale/targoviste/n-a-vazut-mai-mare-stop-judet-trei-tineri-ajuns-spital1_50ad781c7c42d5a66395c02b/index.html 
7. V. Oldenborgh, System and Method for distributed data network having a dynamic topology of communicating a plurality of production nodes with a plurality of consumer nodes without intermediate node logically, (2006)

8. http://digital.ni.com/public.nsf/allkb/862567530005F09C862566BE004E469D

9. Road vehicles - Diagnostics systems-Part2. CARB requirements for interchange of digital information, ISO 9141-2, First Edition (1994)

10. http://www.hoperf.com/upload/rf/RFM12B.pdf 